\title{
Kirche als Kommunikation ${ }^{1}$
}

\author{
von Franz-Josef Eilers
}

Das II. Vatikanische Konzil hat für die Missionsarbeit der Kirche eine wichtige Klärung gebracht: Mission ist nicht das Werk einiger Spezialisten, eben der Missionare, die von allen unterstützt werden müssen. Gründend auf der Kirchenkonstitution des Konzils („Lumen Gentium“) erklärt das Missionsdekret „Ad Gentes“: Mission ist das Wesen der Kirche („Ecclesia peregrinans natura sua missionaria est") - "Die pilgernde Kirche ist ihrem Wesen nach 'missionarisch', da sie selbst ihren Ursprung aus der Sendung des Sohnes und der Sendung des Heiligen Geistes herleitet gemäß dem Plan Gottes, des Vaters“ (AG 2). Die Missionstätigkeit der Kirche gründet in der Inkarnation Jesu Christi: „Was aber vom Herrn ein für allemal verkündet oder in ihm für das Heil des Menschengeschlechtes getan worden ist, muß ausgerufen und ausgesät werden bis ans Ende der Erde. ... So soll, was einmal für alle zum Heil vollzogen worden ist, in allem im Ablauf der Zeiten seine Wirkung erlangen ..." (AG 3). Die Kirche setzt diese Sendung Christi selbst fort.

Für die Kommunikation der Kirche steht bisher eine solche klare Feststellung aus. Parallel zum Missionsdekret muß man auch sagen, daß die pilgernde Kirche ihrem Wesen nach Kommunikation ist: Die innertrinitarische Kommunikation Gottes zwischen Vater, Sohn und Heiligem Geist wird auf den Menschen übertragen, der auch im Blick auf diese Kommunikation nach „seinem Bild und Gleichnis" (Gen 1,27) geschaffen wurde. In der Selbstmitteilung Gottes im Alten Testament und in der Menschwerdung Jesu Christi (im Neuen Testament) kommuniziert Gott mit dem Menschen als Kommunikationspartner. In der Sendung des Heiligen Geistes wird diese Kommunikation Gottes an die Menschen dann als das Wesen der Kirche herausgestellt: „Die Sendung der Kirche vollzieht sich mithin durch das Wirken, kraft dessen sie im Gehorsam gegen Christi Gebot getrieben von der Gnade und Liebe des Heiligen Geistes allen Menschen und Völkern in voller Wirklichkeit gegenwärtig wird, um sie durch das Zeugnis des Lebenns, die Verkündigung, die Sakramente und die übrigen Mitteilungsweisen der Gnade zum Glauben, zur Freiheit und zum Frieden Christi zu führen ...“ (AG 5).

Kirchliche Dokumente zur Kommunikation haben offensichtlich bis heute diese grundlegende Tatsache nicht genügend herausgestellt und vor allem nicht zur Grundlage aller weiteren Überlegungen gemacht.

Auch die neue Pastoralinstruktion "Aetatis Novae" vom 22. Februar 1992 bleibt weit hinter dieser theologischen Wirklichkeit zurück, wenn es dort einfach heißt: „Die Kommunikation muß der kirchlichen Gemeinschaft am Herzen

Franz-Josef Eilers svd ist Professor und geistlicher Berater am Divine Word Seminary, Tagaytay City/Philippinen und Lehrbeauftragter am "Centro Interdisciplinare Sulla Comunicazione Sociale" der Gregoriana/Rom. 
liegen“ (Nr. 6). Ähnlich wie vor dem Konzil bei der Missionsarbeit wird hier immer noch die Kommunikation der Kirche als eine ihrer Tätigkeiten gesehen, aber nicht als das Wesen der Kirche, als Fortsetzung der kommunikativen Sendung Jesu Christi. Die Kommunikation der Kirche gründet in ihrem Wesen. Kirche ist Kommunikation: Ecclesia peregrinans natura sua est communicativa! Wie könnte die Kirche ihre Aufgabe als Frohe Botschaft anders erfüllen?

Offensichtlich wird diese Tatsache der Kirche als Kommunikation immer wieder durch ein einseitiges Medienverständnis beeinträchtigt. Die Wirklichkeit der Kirche ist wesentlich mehr als medial und vor allem als massen-medial! Die Päpstliche Kommission (jetzt: Rat) war schon als Kommission für die Instrumente der sozialen Kommunikation gegründet worden und die neue Pastoralinstruktion ist völlig an dieser Medienorientierung ausgerichtet, wenn es z. B. geht um ,... die Medien im Dienst des Menschen und der Kulturen .. im Dienst der kirchlichen Gemeinschaft .. im Dienst einer Neuevangelisierung“ (Nr. 7-11). Unter 'Medien' werden hier im wesentlichen die Massenmedien verstanden.

Wenn man diese Fixierung auf die Medien verlassen und sich mehr auf den Begriff der 'Kommunikation der Kirche' gründen würde, käme man der theologischen und praktischen Wirklichkeit wohl wesentlich näher. Kirche ist aus ihrem Wesen her Kommunikation und was immer sie ist und tut, vergegenwärtigt, kommuniziert Gottes Wirklichkeit. Die Kommunikation der Kirche ist wesentlich mehr als nur der Gebrauch dieser oder jener technischen Mittel. Was Papst Johannes Paul II. in "Rei Sollicitudo Socialis“ (Nr. 28) für den Besitzstand der Entwicklungsländer sagt, gilt mutatis mutandis auch hier: es ist nicht das Haben, sondern das Sein, welches entscheidet. Das Sein der Kirche aber findet seinen Ausdruck in vielerlei Formen der Kommunikation, angefangen vom Verhalten der Kirchenvertreter und der Kirchenmitglieder ( ZZeugnis des Lebens“) über die Gestaltung und kommunikative Dimension der Gottesdienste (nicht nur in Radio oder Fernsehen!), der Predigt und Verkündigung (Homiletik, Katechetik) bis zum öffentlichen Bild („Image") der kirchlichen Gemeinschaft. Kirche als solche ist kommunkatives Geschehen und muß als solches ernst genommen werden. Vor der Frage des Mediengebrauchs („Medien im Dienst von ..."), des Rechts auf Massenmedien und deren Anwendung steht die Tatsache des kommunikativen Seins der Kirche, das sich auch in ihren Mitgliedern verwirklicht.

Die Vorbereitungskommission für das Kommunikationsdekret des II. Vatikanums hat in einer Fußnote vorgeschlagen, Ausdrücke wie Massenmedien, Distributionsmedien o. ä. zu vermeiden und sie durch einen neuen Ausdruck für den kirchlichen Bereich zu ersetzen, weil diese herkömmlichen Begriffe für die kirchliche Auffassung einer sozialen Kommunikation nicht ausreichend seien. Der als "soziale Kommunikation" vorgeschlagene neue Begriff ist zwar in den kirchlichen Sprachgebrauch - und darüber hinaus auch in die säkulare Sprache vor allem lateinisch-sprachiger Länder - aufgenommen worden, wurde aber weder vom Konzil noch sonst weiter diskutiert und erklärt. Statt die Gelegenheit einer gründlicheren Klärung zu nutzen, fällt auch die neue Pastoralinstruktion auf den schon von der Vorbereitungskommission des Vatikanums kritisierten Gebrauch der 'Medien' zurück. 
Statt einem breiten Verständnis von Kommunikation in menschlicher Gesellschaft den Weg zu bahnen, begrenzt man sich wie auch bereits 1987 in den Leitlinien für die Kommunikationsausbildung künftiger Priester wieder auf Kommunikation als technisch-medialen Vorgang, was praktisch eine Begrenzung auf Massenmedien bedeutet. Dabei wird nicht genügend berücksichtigt, daß gesellschaftliche ( ,soziale“) Kommunikation ja bereits mit der intrapersonalen, vor allem aber mit der interpersonalen Kommunikation beginnt, die über die Gruppenkommunikation dann schließlich auch zur Massenkommunikation führt. Massenmedien sind in dieser Sicht nur ein Teil der gesamtgesellschaftlichen Kommunikation. Eine solche umfassende Sicht würde auch mehr die Kirche als Kommunikation in der gesamten Breite sehen, wie es etwa die Apostolische Exhortation „Evangelii Nuntiandi“ deutlich macht, wo von sieben Mitteln der Evangelisation die Massenmedien nur eines sind (Nr. 40-48).

Die neue Pastoralinstruktion weist zwar unter Nr. 11 zaghaft auf eine solche Möglichkeit hin, ohne aber die Quelle zu nennen, oder auch in eine klarere Entwicklung zu gehen. Unter dieser Perspektive der 'sozialen Kommunikation' ist zwar der Hinweis auf die "volkstümlichen Medien" oder anderer traditioneller Ausdrucksformen (Nr. 16) zu begrüßen, deren Klassifizierung als "alternative Medien (!)“ schränkt ihren Stellenwert gegenüber der ${ }_{n}$ Allgegenwart der Massenmedien“ jedoch sofort stark ein. Statt defensiv die Massenmedien auf der einen Seite als Bedrohung von Moral und Kirche zu sehen (z. B. Nr. 4, 5, 7, 12-15) oder vor einem gewissen Medienoptimismus zu erlahmen (z. B. Nr. 1, 4, 8, 18-19), sollte man sich auf die Grundtatsache einer Kirche als Kommunikation und/oder der Kirche als eines kommunikativen Geschehens in allen Kommunikationsdimensionen menschlicher Gesellschaft besinnen.

Es ist nicht das Haben und Besitzen oder Beherrschen der Medien, was an erster Stelle entscheidet, sondern das Sein der Kirche als kommunikativer Institution, Person und Verpflichtung, die sich auch im Leben des Einzelnen und entsprechender Gemeinschaften verwirklicht. Eine solche Sicht hat dann auch Folgen etwa für eine Ausbildung in Kommunikation, die zwar generell in Nr. 18 der neuen Pastoralinstruktion angesprochen wird, ohne etwa auf Nr. 111 von 'Communio et Progressio' zu verweisen, wo die Dinge bereits wesentlich klarer und detaillierter gesagt wurden. Mit Generalisierungen, wie etwa der Feststellung, daß überall die Menschen heute unter dem Einfluß der Medien ständen (Nr. 1) oder Wiederholungen von Dingen, die in früheren oder neueren anderen kirchlichen Dokumenten, wie z. B. in 'Christifideles Laici' oder 'Redemptoris Missio' wesentlich klarer und ausführlicher gesagt wurden (z. B. Nr. 6, 8 15, 18, 20 ) ist dem Anliegen echter sozialer Kommunikation und der Kirche als Kommunikation nur wenig gedient.

In der Nachfolge von 'Communio et Progressio', der ersten vom Konzil beauftragten Pastoralinstruktion (1971), hat die damalige Päpstliche Kommission für die sozialen Kommunikationsmittel unter ihrem rührigen Präsidenten, dem jetzigen Kardinal Andreas Deskur kontinentale Kommunikationskonferenzen auf der Ebene der Bischofskonferenzen in Lateinamerika, Afrika, Asien, Ozeanien und später auch Europa gehalten. Manches aus den Resolutionen dieser Konferenzen wäre für eine neue Kommunikationspastoral nützlich gewe- 
sen und hätte wahrscheinlich vor einer zu sehr westlich - massenmedialen Sicht bewahrt. Dies muß vor allem auch im Blick auf die wachsende Bedeutung von Lateinamerika, Afrika und Asien für die Zukunft der Kirche in der Welt gesagt werden.

Europa und Nordamerika sollten nicht mehr allein der Maßstab für eine kommunikative Kirche sein, die sich von ihrer Mediengläubigkeit - sei es nun Medienoptimismus oder Medienpessimismus - lösen und auf die theologischen und missionarischen Grundlagen besinnen sollte. Von einer solchen Grundlage ergäben sich auch andere Antworten für alle jene, die Kulturen und ihre kommunikativen Ausdrucksformen ernst nehmen möchten.

Kommunikation ist gerade nicht ein Instrument, der Besitz oder Gebrauch eines (Massen-)Mediums, einer Methode, sondern ein Sein, das Gottes Liebe unter den Menschen - auch im Sinne vom 'Communio et Progressio' Nr. 11: Jesus Christus als vollkommener Kommunikator - nicht nur verkündet und vermittelt, sondern zuerst lebt. Kirche als Kommunikation ist eine kommunikative Gegenwart Jesu Christi unter und mit den Menschen aller Kulturen und aller Zeiten, auch der unsrigen des kommenden dritten Jahrtausends.

\section{Anmerkung:}

1 Vgl. meinen Artikel zum Begriff "sozialer Kommunikation“, in: CS 20 (1987) 1-10; vgl. im folgenden auch: F. J. Eilers, Communicating between Cultures, Manila 1992, bes. 145-150. 